\title{
Aquisição dos gestos na comunicação pré-linguística: uma abordagem teórica
}

\author{
Acquisition of gestures in prelinguistic communication: a \\ theoretical approach
}

\author{
Etelvina do Rosário Silva Lima ${ }^{1}$, Anabela Cruz-Santos ${ }^{2}$
}

\begin{abstract}
RESUMO
Com o objetivo de compreender as relações entre o gesto e a linguagem e a relevância do gesto no desenvolvimento de competências comunicativo-linguísticas foi realizada uma pesquisa da literatura nacional e internacional. A análise dos dados de múltiplas áreas científicas indica influências transversais do gesto natural na evolução da competência comunicativo-linguística do Homem. Assim, a identificação do sistema de neurónios-espelho promoveu o emergir de vários trabalhos que levantaram hipóteses sobre o paralelismo entre a evolução do gesto versus a evolução da linguagem. A literatura aponta que os gestos naturais possuem uma força preditiva no desenvolvimento da linguagem e ao longo de diferentes períodos influencia diversas componentes: pragmática, semântica e morfossintaxe. A influência do gesto natural para a competência comunicativa continua ao longo da vida, visto que o uso de gestos naturais simultâneos ao discurso possuem a função de desambiguar o conteúdo da mensagem para o interlocutor e de organizar o discurso ao emissor. O uso dos gestos naturais na promoção de competências comunicativo-linguísticas em crianças com perturbações da comunicação pré-linguística torna-se incontornável mas requer um aprofundamento de conhecimentos dado que os estudos relacionados com as competências pragmáticas iniciais direcionam-se sobretudo com o gesto de apontar. Outros estudos analisaram os gestos naturais na compreensão das relações com as componentes da semântica e morfossintaxe em idades mais tardias.
\end{abstract}

Descritores: Gestos; Comunicação não verbal; Linguagem; Surdez; Transtornos da comunicação

\section{INTRODUÇÃO}

Os gestos naturais constituem a primeira ferramenta de comunicação simbólica. A sua função vai sendo transformada ao longo dos períodos de evolução da comunicação mas que suporta a nossa eficácia comunicativa ao longo da vida. Estes gestos são considerados a primeira forma de comunicação simbólica. Embora não sejam convencionados ou símbolos arbitrários como as palavras são, eles ocorrem repetidamente na mesma forma física. Inicialmente, a sua força comunicativa está presa ao contexto onde ocorrem mas o seu uso vai conferindo um significado cada vez mais independente do contexto e mais ligado ao próprio gesto.

A verdade é que apesar de os bebés vocalizarem desde o nascimento, é através dos gestos que as crianças veiculam

Trabalho realizado no âmbito de um Projeto de Doutoramento em Estudos da Criança, na especialidade de Educação Especial, Universidade do Minho - Braga, Portugal.

Conflito de interesses: Não

(1) Instituto Politécnico de Leiria, Universidade do Minho - Braga, Portugal.

(2) Instituto de Educação, Universidade do Minho - Braga, Portugal.

Endereço para correspondência: Etelvina Lima. Escola Superior de Saúde, Instituto Politécnico de Leiria. E-mail: etelvina.lima@ipleiria.pt

Recebido em: 25/6/2012; Aceito em: 10/10/2012 e estruturam as suas primeiras intenções comunicativas. Os gestos naturais são a pedra basilar na construção da linguagem dado que representam a forma facilitada da criança iniciar a construção de conceitos por um lado e é a alternativa para exprimir conceitos quando ainda não dominam a modalidade verbal com todas as regras que estão inerentes à utilização de uma língua ${ }^{(1-4)}$. Os gestos contribuem para o desenvolvimento de símbolos e desbravam o caminho para a linguagem falada.

Linhas de investigação têm apresentado evidências que fortalecem a possibilidade dos gestos terem sido a primeira forma de comunicação e que com base neles desenvolve-se a linguagem, através de um processo de lento evolução neurológico, social e cognitivo ${ }^{(1,5-9)}$. Similarmente, o processo ontogénico identifica o uso de gestos naturais como fator preditivo da emergência da linguagem ${ }^{(2,10-14)}$.

$\mathrm{Na}$ intervenção precoce de crianças com perturbações da linguagem este cômputo de evidências científicas torna incontornável a importância de centralizar o uso dos gestos naturais em programas direcionados para a promoção de competências comunicativo-linguísticas. Ainda que nos últimos anos tenha crescido o interesse no uso dos gestos simbólicos ${ }^{(11,15,16)}$ na prevenção e intervenção de crianças em risco, os gestos naturais são mais abrangentes e vastos que o referido grupo de gestos $^{(17,18)}$. 
Torna-se, então, necessário reunir e integrar as informações que advêm de várias áreas do saber para compreender os gestos naturais de forma holística e abrangente. Só será possível processar as influências e particularidades do gesto natural perspetivando a sua transversalidade na comunicação.

\section{REVISÃO DA LITERATURA}

A revisão da literatura que se segue é resultado da pesquisa realizada nos acervos bibliográficos de várias bibliotecas nomeadamente, na Biblioteca da Universidade do Minho, Biblioteca da Faculdade de Psicologia e Ciências da Educação da Universidade do Porto, Biblioteca da Escola Superior de Saúde de Alcoitão e Biblioteca do Instituto Politécnico de Leiria.

Ainda, foi realizada uma pesquisa sistemática nas seguintes bases de dados eletrónicas: ScienceDirect, PubMed, Scopus, Lilacs e SciELO. Inicialmente foram utilizadas, de forma sistemática em todos os acervos, várias combinações de palavras-chave. Optou-se por fazer a pesquisa em Português através da combinação das palavras-chave: gestos; gestos naturais; pré-linguagem, e; comunicação. Em Inglês fez-se uso das palavras-chave: gestures; communication; communicative function; early language, e; prelinguistic. Atingida a saturação reconhecida pela repetição de estudos acrescentou-se palavras-chave em Inglês (deafness, hearing impairment e signs) e em Português (surdez, deficiência auditiva e língua gestual).

Do cômputo da informação teórica recolhida, este artigo aborda o que se sabe sobre a influência do uso gesto no desenvolvimento da comunicação. Dada a variedade de gestos naturais identificados na literatura, torna-se pertinente nomear e definir diferentes tipos de gestos.

\section{Terminologia do gesto}

São inúmeros os termos utilizados para referir diferentes tipos de gestos. Este fenómeno acontece porque à medida que a criança evoluiu nas funções comunicativas veiculadas por um determinado gesto num determinado período do seu desenvolvimento comunicativo, diferentes autores optam por diferentes nomenclaturas e definições. Nesta seção encontram-se as posições de diferentes autores selecionados com o intuito demonstrar a variedade de nomenclaturas versus semelhanças e paralelismos.

É possível identificar quatro tipos de gestos utilizados antes dos dez meses de idade ${ }^{(19)}$ : os gestos expressivos, utilizados para expressar emoções (e.g. bater palmas, bater com os pés no chão); os gestos instrumentais, usados para controlar o comportamento do outro (e.g. estender os braços para pedir colo, abrir e fechar a mão para tentar agarrar um objeto enquanto alterna o contacto ocular entre o interlocutor e o objeto); os gestos enactivos*, que representam ações com algum nível de simbolismo (e.g. mão ao lado da cara representando a ação "dormir"); os gestos deíticos, utilizados para mostrar aos outros os objetos (e.g. apontar) $)^{(21)}$.

Outra classificação indica três tipos de gestos que as crian- ças usam durante as suas primeiras incursões comunicativas: os gestos rituais, os gestos deíticos e os gestos simbólicos ou referenciais ${ }^{(4)}$.

Os gestos rituais são aqueles que a criança utiliza para que se faça algo. Estes gestos são transversais à maioria das populações porque são gestos desenvolvidos a partir de uma ação motora que à medida que vai tendo uma resposta social a criança acaba por resumir o movimento apenas a um gesto. Por exemplo, o gesto de fechar a mão estendida usado pelas crianças para pedir algo é transversal a várias culturas distribuídas por vários pontos do globo. Inicialmente as crianças realizam os movimentos repetidos de abrir e fechar as mãos na tentativa de agarrar um objeto mas á medida que ela vai obtendo uma consequência em resposta ao seu movimento (o adulto dá-lhe o objeto) tende a reduzir o gesto realizado em direção ao outro, até estruturar uma versão estilizada do movimento (o gesto ritual) veiculado com intencionalidade comunicativa.

Este processo de aprendizagem não depende da observação do comportamento do outro para posterior imitação, não sendo por uma aprendizagem cultural que dependa da compreensão das intenções comunicativas do outro. Assim os gestos rituais distinguem-se dos gestos simbólicos porque a criança não está a influenciar a atenção do outro através de um ato comunicativo mutuamente compreendido mas apenas para atingir um resultado concreto ${ }^{(4)}$.

Estes gestos parecem estar mais associados a funções comunicativas adquiridas mais precocemente, relacionadas com a satisfação das necessidades.

Os gestos deíticos são definidos como gestos desenvolvidos para direcionar a atenção do adulto para entidade exteriores, sendo o apontar um gesto protótipo deste tipo de gestos. Dependendo do processo de aprendizagem associado à aquisição deste gesto (apontar) ele pode ser semelhante aos gestos ritualizados ou não.

A criança pode adquirir o gesto pelo mesmo processo descrito anteriormente, ou pode adquiri-lo imitando o adulto e utilizando-o para veicular o mesmo tipo de funções comunicativas como, por exemplo, o comentar algo. Nesse caso a criança não utiliza o gesto apenas para obter algo estando patente uma troca comunicativa em que é necessário aceder ao estado mental do outro.

Os gestos simbólicos são utilizados em atos comunicativos que estão associados a um referente metonímico ou icónico ${ }^{(4)}$. Este tipo de gestos são construídos com base a características do objeto ou ação que representam, como por exemplo, estender os braços ao lado do corpo para referir "avião" ou apontar com o polegar estendido em frente à boca aberta para referir "beber".

A iconicidade dos gestos é apenas percecionada pelos adultos, pelo que estes gestos são adquiridos através da imitação em trocas comunicativas com os adultos. Efetivamente, alguns estudos desenvolvidos na área evidenciam que; a iconicidade dos gestos não facilita a sua aquisição por crianças com surdez nos momentos iniciais da aquisição; no segundo ano de vida as crianças aprendem gestos arbitrários com tanta facilidade

\footnotetext{
* Nomenclatura inspirada em trabalhos no âmbito do desenvolvimento cognitivo em que se designava o primeiro nível de representação enactiva, aquele em que as crianças representariam o mundo através das suas ações sensório-motoras imitando, manipulando e agindo sobre objetos ${ }^{(20)}$.
} 
como aprendem palavras; as crianças aos 18 meses de idade não conseguem entender intenções comunicativas específicas veiculadas por elementos icónicos ${ }^{(4)}$.

Num estudo que visava compreender o processo pelo qual as crianças transformavam os gestos naturais em linguagem foram categorizados os gestos produzidos pelas crianças e pelas suas mães, em três grupos ${ }^{(14)}$ :

- Gestos deíticos usados para indicar objetos, pessoas e locais no contexto imediato e com significados ligados ao contexto. Observaram a produção de gestos em que situando um objeto no campo de visão do par comunicativo era realizado o gesto de apontar para o mesmo objeto com o dedo ou com a palma da mão;

- Gestos convencionais incluíam movimentos do corpo e das mãos, por exemplo, estender a palma da mão para solicitar um objeto ("dar"), colocar uma ou as duas mãos num lado da cara, inclinando a cabeça ("sono/dormir"), acenar com a cabeça ("sim"), virar e levantar as palmas para cima ("não sei").

- Gestos icónicos para expressar ações ou atributos de referências concretas, através de movimentos corporais ou manuais, por exemplo, movendo o dedo indicador em círculos para indicar os movimentos de uma bola, ou colocando duas palmas vertical sobre a cabeça para indicar a forma das orelhas de um coelho.

Relativamente à classificação de gestos simultâneos ao discurso veiculados por movimentos manuais funcionais enumeram-se cinco tipos de gestos $^{(22)}$ :

- Gestos deíticos frequentemente originados pela funcionalidade de agarrar objetos numa localização espacial particular;

- Gestos copulares em que a mão move-se em direção à boca representando levar comida à boca e referenciar "comer";

- Gestos metafóricos em que as mãos se movem para representar a manipulação dos objetos, e.g., pressionar as mãos em forma de garra para referir "amachucar";

- Gestos espásticos que são realizados movimentos de batimentos rítmicos para referenciar por exemplo, o andar;

- Gestos aversivos referindo-se a gestos em que as mãos adotam uma postura defensiva da cabeça ou do corpo.

\section{Neurofisiologia do gesto}

A teoria biogenética sobre a recapitulação estabelece que a ontogenia recapitula a filogenia. Nesta premissa podemos determinar o mesmo paralelismo entre o gesto e a evolução da linguagem e o processo de aquisição e desenvolvimento da linguagem.

A literatura tem vindo a apontar relações entre o padrão de desenvolvimento da comunicação nas crianças e algumas evidências que apontam para padrões similares no processo evolutivo da Humanidade que permitiu a emergência da comunicação e linguagem, fator determinante para a competência social do Homem e caraterística distintiva de todas as outras espécies $^{(4,6)}$. A perspetiva filogenética, que a origem das línguas gestuais é coincidente com a origem das línguas humanas, remonta às primeiras discussões filosóficas sobre o início da linguagem na Humanidade ${ }^{(1,22)}$.
Existem indicações ao longo da evolução da espécie o sistema de neurónios-espelho tenha desempenhado um papel preponderante no aumento da complexidade da comunicação, estando a emergência do sistema comunicativo-linguístico utilizado atualmente ${ }^{(5,23)}$ ligado a múltiplas ligações entre a ação, o gesto, o significado e a linguagem ${ }^{(5,7,8,22,24-26)}$. Este sistema é ativado quando é desempenhada uma ação com ou a boca ou uma ação manual relacionada com um objeto. Ainda, é ativada despertando a atenção para se observar o outro a realizar ações semelhantes ${ }^{(7,22,26)}$ permitindo a imitação das mesmas.

Os neurónios-espelho do córtex pré-motor e parietal, além de codificarem o objetivo de uma ação motora, também codificam a intenção subjacente a essa mesma ação, possibilitando a codificação da ação de pegar um objeto mas também de agarrar um objeto com a intenção de o comer ${ }^{(26)}$.

Os estudos de neuro-imagem e estimulação elétrica transcraniana mostram que as respostas sobre as relações entre os gestos e as palavras encontram-se na área de Broca ${ }^{(11)}$. Esta área cerebral está envolvida no sistema de neurónios-espelho, sendo ativada para o reconhecimento de ações manuais e orais $^{(26)}$ e para a compreensão dos significados presentes nos gestos comunicativos e na articulação dos sons/palavras ${ }^{(8)}$. Efetivamente, as palavras e os gestos manuais que partilham significado influenciam-se mutuamente quando são produzidos simultaneamente.

Dado que o sistema de neurónios espelho é dinâmico, ativa diferentes áreas de acordo com a ação que está a ser observada. Se o objetivo for compreender a mensagem, veiculada num ato comunicativo, que envolva os gestos associados ao discurso, serão ativadas áreas do sistema de neurónios-espelho diferentes do que se o objetivo pretendido for entender o discurso tendo presente a informação visual dos movimentos orais ${ }^{(8)}$.

As explicações sobre a natureza e os processos destas interações são melhor explicados em estudos comportamentais que avançam com a hipótese da integração entre o gesto e a palavra ocorrer pela transformação da intenção social, em interagir diretamente com o interlocutor, que em diferentes graus de complexidade necessita de fazer uso desde gestos até a códigos orais convencionais ${ }^{(9,11)}$.

\section{O gesto e a linguagem: interligações}

O uso dos gestos comunicativos são preditores do desenvolvimento linguístico em duas vertentes, pois por um lado existe uma relação de grandeza entre a quantidade de objetos que o bebé aponta e a compreensão de vocabulário que possuirá no futuro próximo, e por outro lado dá indicação de quais as palavras que a criança irá adquirir em breve (e.g. se aponta para o cão e não para o osso, a probabilidade é que a criança venha a adquirir a palavra cão antes de $o s s o)^{(3,13,27,28)}$.

Verifica-se que os gestos também desempenham um papel preditivo no domínio do desenvolvimento sintático, pois a idade em que a criança produz o gesto do apontar + palavra prediz a idade em que produzirá frases de duas palavras, ou seja, primeiro a criança apenas aponta, depois aponta com a palavra papa com a intenção de veicular a mensagem quero comer a bolacha. Mais tarde, construções de duas palavras são predizíveis pela combinação de gesto + palavra, i.e., 
produção da palavra popó simultaneamente ao apontar para o pai, prediz frases do tipo nome+nome, enquanto a palavra comer ao mesmo tempo que aponta para a mãe prediz a frase do tipo verbo+nome ${ }^{(28-30)}$.

Sabe-se que a quantidade de gestos produzidos aos 18 meses prediz o vocabulário utilizado aos 42 meses e que as combinações gesto+palavra também predizem a complexidade sintática aos 42 meses. Salienta-se que só se encontram correlações na direção quantidade de gestos versus número de vocabulário ou combinações gesto + palavra vs complexidade sintática $^{(28)}$. Assim, as crianças que utilizam uma grande quantidade de gestos aos 18 meses podem ter menor desempenho na produção de frases sintaticamente complexas aos 42 meses.

Estes dados indicam que podemos prever as caraterísticas do padrão linguístico de uma criança, quanto às componentes linguísticas (semântica e sintaxe), com dois anos de antecedência, apenas através da observação do uso dos gestos.

Indica-se três possibilidades de mecanismos subjacentes às relações entre o uso dos gestos e o desenvolvimento da linguagem: a) o uso dos gestos pode ser um indicador do desenvolvimento do interesse comunicativo por parte dos bebés e o tipo de significado que eles escolhem veicular, que mais tarde virá a ser revelado na linguagem; b) os gestos dão ao bebé um modo de entrar na interação comunicativa antes de ter níveis de desenvolvimento da linguagem que suportem as experiências comunicativas (contudo a forma como este processo se desenrola está por explicar), ou/e; c) os gestos das crianças induzem a linguagem verbal dos outros, sendo esta possibilidade que melhor explica a estreita relação entre os conteúdos dos gestos da criança e o conteúdo da linguagem subsequente ${ }^{(31)}$.

Relativamente a esta hipótese um estudo analisou as respostas das mães aos gestos naturais que os seus filhos usavam para se exprimir ${ }^{(32)}$. Verificou-se que as mães traduzem esses gestos em palavras. As mães traduzem os gestos dos bebés em palavras ou frases promovendo a relação entre o que o bebé pensa e como a linguagem pode expressar esse pensamento. $\mathrm{Na}$ verdade, há indicadores de que os gestos desempenham um papel duplo no processo de desenvolvimento da linguagem. Por um lado, eles são preditores do desenvolvimento e por outro, o seu uso suporta o desenvolvimento.

Um estudo que analisou o uso de gestos privados, i.e., não dirigidos ao outro, distinguiu dois tipos: gestos ostensivos privados e gestos de apontar privados $^{(33)}$. Verificou-se que as crianças os utilizavam de forma autorreflexiva para se regularem e para serem auxiliares na forma como construíam e organizavam o seu pensamento.

Através da análise do sistema de comunicação gestual desenvolvido por crianças surdas (privadas do acesso tanto à língua nativa gestual como à oralista), encontrou-se uma série de características comuns a todos os outros sistemas linguísticos utilizados pelos humanos, as quais se denominou, propriedades resilientes da linguagem ${ }^{(34)}$. Verificou-se que quando o gesto é utilizado com o objetivo de desempenhar todas as funções comunicativas dos atos de fala, este assume propriedades semelhantes ao do discurso ao nível das características da palavra e da frase. Poder-se-ia inferir então que, o uso do gesto nas interações das crianças ajudam-na a estruturar e adquirir funções comunicativas? Esta inferência não pode ser linear. Quando os atos de fala e os gestos são utilizados em conjunto, o gesto adquire uma função complementar e desorganiza-se, perdendo as propriedades de resiliência do discurso. Só na ausência de atos de fala os gestos adquirem o papel mais relevante e estruturam-se com as referidas propriedades.

Num estudo em que pediu a adultos para descrever as cenas de uma gravação audiovisual, verificou-se que quando os adultos utilizavam o gesto com a linguagem oral, raramente combinavam o gesto em cadeia de forma consistente e orde$\operatorname{nada}^{(35)}$. No entanto, quando impedidos de utilizar a linguagem oral, desenvolviam no próprio momento uma forma de comunicação com gestos combinados em cadeias ordenadas, cuja ordem diferia da ordem das palavras da sua língua materna.

Os estudos realizados nesta área são importantes ferramentas para que os investigadores entendam o processo realizado pelas crianças para adquirir linguagem quer em condições típicas, quer atípicas, como no caso da surdez ou nos casos em que surgem perturbações da linguagem.

Quando as crianças apresentam problemas em transmitir oralmente a sua mensagem, o meio funcional para veicularem as suas mensagens é através dos gestos. Na realidade, todas as crianças usam os gestos naturais, quer estejam no início da aquisição linguística quer no início da aquisição de uma segunda língua ${ }^{(36)}$

As crianças de idades precoces referem metade dos objetos apenas com gestos naturais (maioritariamente o apontar) suportados no contexto, contrastando com uma pequena percentagem de referências suportadas pelas palavras ou pelas palavras acompanhadas com os gestos ${ }^{(37)}$. O facto de a criança apontar para um objeto será preditor da aprendizagem dessa mesma palavra quer seja pelo facto do gesto despoletar a nomeação do objeto por parte do outro, quer seja, porque indica o interesse da criança por este mesmo objeto. Numa análise do desenvolvimento do gesto demonstrou-se que em diferentes sessões as crianças raramente referiam-se oralmente a um objeto sem o referir antes com o apontar ${ }^{(37)}$. Cerca de $80 \%$ das vezes, as primeiras referências de uma criança a um referente/significado/conceito foram expressas através de gestos, sendo mais tarde referenciadas pelo gesto e palavra, simultaneamente, e só mais tarde pela palavra.

Evidentemente, esta função de suporte da aquisição lexical vai sendo gradualmente abandonada dado que os adultos aprendem novo léxico sem que seja necessário este processo. $\mathrm{O}$ que se verifica é que este processo só está presente mesmo no início do desenrolar do processo de aquisição de competências comunicativas. Por volta dos 26 meses, em que as crianças dão os primeiros passos no período linguístico as novas entradas lexicais são realizadas diretamente pela palavra ${ }^{(16)}$.

Estas indicações vão de encontro às premissas teóricas das abordagens sócio-cognitivas. $\mathrm{O}$ fato deste estudo não ter verificado uma grande influência de fatores do meio neste binómio apontar/desenvolvimento da linguagem, deverá estar mais relacionado com falta de dados do que a efetiva inexistência de relação de fatores sociais, dado que a compreensão e produção do gesto do apontar é o primeiro indicador das noções de intersubjetividade secundária, i.e., compreender os outros como agentes intencionais. 
O apontar é um marco único no desenvolvimento social e linguístico das crianças presente desde os 12 meses de idade ${ }^{(38)}$. Um estudo meta-analítico mostrou uma forte correlação entre o gesto do apontar e a emergência da linguagem relatando uma inequívoca relação entre o apontar e o desenvolvimento de competências na compreensão e expressão da linguagem, sendo ainda mais significativas quando o gesto é produzido num ato comunicativo declarativo ${ }^{(2)}$.

Mas as crianças não usam apenas o gesto natural do apontar, entre muitos outros elas usam os gestos icónicos que são gestos que não dependem tanto do contexto, pois são construídos com base em aspetos do seu referente (e.g., movimento repetido de colocar as mãos debaixo das axilas para representar os macacos). Por esta mesma razão eles são muito aproximados e mais estreitamente ligados com as características das palavras. Na verdade, muitas vezes suportam a comunicação da mesma forma que as palavras verbais orais o fazem.

O facto é que as crianças utilizam os gestos para desempenhar várias funções comunicativas, designadamente, podem utilizar para pedir objetos ou ações, para informar (e.g., "não há" no caso da não existência/existência), para comentar (e.g., "grande" no caso dos atributos dos objetos), para recusar (e.g., "não" no caso de rejeitar uma ação).

Efetivamente, os gestos naturais fazem parte do nosso quotidiano permanecendo na idade adulta, desempenhando funções diferentes de acordo com a situação em que são utilizados.

Existe um efeito do gesto na compreensão do discurso em adultos, dado que nas situações em que as condições comunicativas estão dificultadas, quer por ruído (condição externa) quer por surdez (condição interna), a informação visual incorpora-se no processamento do discurso ${ }^{(39)}$. As interferências externas conduzem à inclusão das pistas gestuais num período finito no tempo, enquanto as internas conduzem a um uso permanente da informação gestual, possivelmente provocado por modificações da na distribuição espacial da atenção visual. Os indivíduos com surdez tendem a usar a informação visual como uma estratégia para facilitar a compreensão do discurso, pelo que os gestos naturais que acompanham a fala são uma ferramenta fundamental na compreensão do discurso das situações quotidianas.

Nos primórdios da comunicação a condição da surdez não parece produzir diferenças no uso dos gestos naturais, pelo que, tanto as crianças ouvintes e surdas usam-nos para comunicar.

Não existem diferenças relevantes no padrão comunicativo de crianças ouvintes e com surdez (profunda e severa) que possuíam um léxico menor ou igual a dez palavras, no seu segundo ano de vida ${ }^{(40)}$. Embora, o uso de palavras espontâneas pelas crianças com surdez seja ligeiramente menor observa-se uma diferença significativa no uso de gestos inovadores por parte das crianças surdas.

Ao longo do desenvolvimento as crianças ouvintes usam o gesto como transição para a linguagem oral, produzindo os gestos naturais para acompanhar as verbalizações, sendo que estas combinações perfeitamente integradas no momento e no significado. Pelo contrário, os gestos naturais nas crianças com surdez emergem de duas formas assumindo funções de suporte natural da comunicação mas também assumindo gradualmente as funções mais características da linguagem convencionada (a língua falada ou língua gestual) ${ }^{(41)}$.

A questão que fica por explicar é se nos primórdios da comunicação, apesar de, tanto as crianças ouvintes como as crianças com surdez utilizarem gestos naturais, (talvez na mesma quantidade) elas usam-nos com as mesmas funções?

Quando existem alterações na aquisição de competências linguísticas as funções alteram-se.

A possibilidade avançada é que passado o período inicial em que o gesto natural suporta a aquisição linguística, ele passa a ter uma função complementar da linguagem.

As crianças com Perturbação Específica do Desenvolvimento da Linguagem** (PEDL) não utilizam mais gestos do que as crianças com desenvolvimento típico, embora as primeiras usem o gesto de forma a contornar as dificuldades que sentem em exprimir a mensagem verbalmente ${ }^{(42)}$.

Quando as crianças experimentam dificuldades em se exprimir linguisticamente o gesto adquire uma forma mais estruturada (não pela quantidade mas pela qualidade do uso dos gestos).

Esta leitura é compatível com as já referidas estratégias utilizadas pelos adultos que utilizando o gesto com a linguagem oral, raramente combinavam o gesto em cadeia de forma consistente e ordenada contrariamente a quando impedidos de utilizar a linguagem oral ${ }^{(35)}$.

Assim, os gestos naturais são, efetivamente, utilizados ao longo da vida para contornar as dificuldades encontradas em expressar ideias em palavras. Este facto pode não ser muito óbvio para o observador porque esta função reside não na quantidade de gestos naturais que são usados mas sim no tipo de informação que é transmitida por gestos.

Efetivamente, as línguas gestuais que sustentam a totalidade da comunicação são muito diferentes dos gestos naturais que acompanham a fala ${ }^{(41)}$.

A verdade é que as línguas gestuais com os gestos codificados são um sistema com todas as características das línguas orais. No entanto, os gestos naturais complementam o discurso oral, pelo que têm funções diferentes, como o de desambiguar a mensagem oral, de enfatizar a mensagem, entre outras. No caso das crianças que se encontram a desenvolver a fase pré-linguística, em que os gestos são naturais/não codificados e emergem para sustentar os atos comunicativos, apresentam-se com maior estrutura.

\section{DISCUSSÃO}

São indubitáveis as interligações entre os gestos naturais e a linguagem. Inúmeras evidências emergem de várias áreas científicas e esclarecem as formas como os gestos facilitam e promovem competência comunicativa tanto no processo de desenvolvimento inicial da comunicação e linguagem como ao longo da nossa vida. A influência do gesto na aquisição de competências comunicativo-linguísticas é transversal à evolução do Homem e do homem. Esse fenómeno de transversalidade, não

\footnotetext{
** Nas Perturbações Específicas do Desenvolvimento da Linguagem observam-se sequências e processos de desenvolvimento diferentes do típico, na ausência de alterações sensoriais, défices emocionais, défices cognitivos ou de perturbações morfológicas ou motoras do sistema fonoarticulatório ${ }^{(43)}$.
} 
se resume à perspetiva filogenética e ontogenética, sendo esta transversalidade longitudinal à competência comunicativo-linguística dos indivíduos.

As contribuições do campo da neurofisiologia na identificação do funcionamento sistema de neurónios-espelho, não só lançam luz sobre aspetos como a capacidade atenção, a capacidade de imitação de ações e gestos, como também explicam como este sistema se interliga com a área de Broca para o desenvolvimento da semântica.

Ainda, estudos comportamentais demonstram a função preditiva do gesto natural no desenvolvimento da linguagem, identificando a sua influência na semântica, na morfossintaxe e na pragmática.

\section{COMENTÁRIOS FINAIS}

Apesar de, nas últimas duas décadas, se reconhecer avan- ços significativos no conhecimento de como o gesto opera na comunicação, a aplicação dos conhecimentos para a promoção de competências comunicativo-linguísticas em crianças com perturbações da comunicação pré-linguística requer dados mais específicos. Os estudos relacionados com as competências pragmáticas iniciais direcionam-se sobretudo com o gesto de apontar e os estudos com os outros grupos de gestos naturais direcionam os seus objetivos para a compreensão das relações com as componentes da semântica e morfossintaxe em idades mais tardias.

Assim, futuros campos de investigação deverão aprofundar o conhecimento sobre a construção de significado e na identificação e compreensão das relações entre as competências pragmáticas iniciais e o uso de um leque abrangente dos gestos naturais.

\begin{abstract}
In order to understand the relationship between gestures and language and the relevance of gestures in the development of communicative and linguistic skills, a review of national and international literature was conducted. The analysis of data from multiple areas of science indicates the cross-influences of gesture in the evolution of the communicative competence of Humankind. The identification of the human mirror system created the background for studies that generated hypotheses about the parallelism between the evolution of gesture versus the evolution of language. Literature indicates that the use of gestures has a predictive power in language development, and different periods of language development influence the components of pragmatics, semantics and morphosyntax. The influence of natural gesture for communicative competence continues throughout life, since co-speech gestures have the purpose to disambiguate the content of the message to the interlocutor and to organize verbal reasoning for the speaker. Using natural gestures in promoting communication and linguistic skills in children with prelinguistic communication disorders has become essential, but it requires deeper knowledge as studies related to the early pragmatic skills are mainly directed towards the pointing gesture. Studies that analyzed other groups of gestures in older children have also contributed for understanding the use of gestures and the development of semantic and morphosyntactic competencies.
\end{abstract}

Keywords: Gestures; Nonverbal communication; Language; Deafness; Communication disorders

\title{
REFERÊNCIAS
}

1. Armstrong DF, Wilcox SE. The gestural origin of language. Oxford: Oxford University Press; 2007.

2. Colonnesi C, Jan JM, Stams G, Koster I, Noom M. The relation between pointing and language development: a meta-analysis. Developmental Review. 2010;30(4):352-66.

3. Iverson JM, Goldin-Meadow S. Gestures paves the way for language development. Psychol Sci. 2005;16(5):368-71.

4. Tomasello M. Constructing a language: a usage-based theory of language acquisition. Cambridge: Harvard University Press; 2003.

5. Arbib MA. From monkey-like action recognition to human language: An evolutionary framework for neurolinguistics. Behav Brian Sci. 2005;28(2):105-67.

6. Cartmill E, Byrne R. Semantics of primate gestures: intencional meanings of orangutan gestures. Anim Cogn. 2010;13(6):793-804.

7. Corballis MC. Mirror neurons and the evolution of language. Brain Lang. 2010;112(1):25-35.

8. Skipper JI, Goldin-Meadow S, Nusbaum HC, Small SL. Speechassociated gestures, Broca's area, and the human mirror system. Brain Lang. 2007;101(3):260-77.
9. Tomasello M, Carpenter M, Call J, Behne T, Moll H. Understanding and sharing intention: The origins of cultural cognition. Behav Brian Sci. 2005;28(5):675-91.

10. Aureli T, Perucchini P, Genco M. Children's understanding of communicative intentions in the middle of the second year of life. Cognitive Development. 2009;24(1):1-12.

11. Barbieri F, Buonocore A, Volta RD, Mauricio G. How symbolic gestures and words interact with each other. Brain Lang. 2009;110(1):1-11.

12. Cochet H, Vauclair J. Pointing gestures produced by toddlers from 15 to 30 months: Different functions, hand shapes and laterality patterns. Infant Behav Dev. 2010;33(4):431-41.

13. Goldin-Meadow S. Using the hands to study how children learn language. In: Colombo J, McCardle P, Freund L, editors. Infants pathways to language. New Jersey: Lawrence Erlbaum Associates; 2009. p. 195-210.

14. Goldin-Meadow S, Mylander C, Franklin A. How children make language out of gesture: Morphological structure in gesture systems developed by American and Chinese deaf children. Cognit Psychol. 2007;55(2):87-135. 
15. Santana A, Guarinello A, Berberian A, Massi G. O estatuto simbólico dos gestos no contexto da surdez. Psicol Estud. 2008;13(2):297-306.

16. Goodwyn S, Acredolo LP. Symbolic gesture versus word: is there a modality advantage for onset of symbol use? Child Dev. 1993;64(3):688-701.

17. Doherty-Sneddon G. The great baby signing debate: Academia meets public interest. Psychologist. 2008;21(4):300-3.

18. Góngora X, Farkas C. Infant sign language program effects on synchronic mother-infant interactions. Infant Behav Dev 2009;32(2):216-25.

19. Nelson K. Making sense: the acquisition of shared meaning. New York: Academic Press; 1985.

20. Bruner J. Realidade mental, mundos possíveis. Porto Alegre: Artes Médicas; 1998.

21. Haynes W. Single word communication: a period of transitions. In: Shulman WH, editor. Communication development: foundations, processes and clinical applications New Jersey: Prentice-Hall; 1994. p. 230-56.

22. Whishaw IQ, Sacrey LA, Travis SG, Gholamrezaei G, Karl JM. The functional origins of speech-related hand gestures. Behav Brian Res. 2010;214(2):206-15.

23. Nagy E, Liotti M, Brown S, Waiter G, Bromiley A, Trevarthen C, et al. The neural mechanisms of reciprocal communication. Brain Res. 2010;1353:159-67.

24. Gentilucci M, Corballis MC. From manual gestures to speech: A gradual transition. Neurosci and Biobehav Rev. 2006;30(7):949-60.

25. Genty E, Breuer T, Hobaiter C, Byrne RW. Gestural communication of the gorilla (Gorilla gorilla): repertoire, intentionality and possible origins. Anim Cogn. 2009;12(3):527-46.

26. Glenberg AM, Gallese V. Action-based language: A theory of language acquisition, comprehension, and production. Cortex. 2012;48(7):905-22

27. Acredolo L, Goodwyn S. Symbolic gesturing in normal infants. Child Dev. 1988;59(2):450-66.

28. Rowe ML, Goldin-Meadow S. Early gesture selectively predicts later language learning. Dev Sci. 2009;12(1):182-7.

29. Iverson J, Capirci O, Volterra V, Goldin-Meadow S. Learning to talk in a gesture-rich world: early communication in Italian vs American children. First Lang. 2008;28(2):164-81.
30. Ozcaliskan S, Goldin-Meadow S. Do parents lead their children by the hand? J Child Lang. 2005;32(3):481-505.

31. Hoff E. Language development. $4^{\text {a }}$ ed. Belmont CA: Wadsworth Publishing; 2008.

32. Goldin-Meadow S, Goodrich W, Sauer E, Iverson J. Young children use their hands to tell their mothers what to say. Dev Sci. 2007;10(6):77885.

33. Rodríguez C, Palacios P. Do private gestures have a self-regulatory function?: a case study. Infant Behav Dev. 2007;30(2):180-94.

34. Goldin-Meadow S, Mylander C. Gestural communication in deaf children: non-effect of parental input on language development. Science. 1983;221(4608):372-4.

35. Gershkoff-Stowe L, Goldin-Meadow S. Is there a natural order for expressing semantic relations? Cogn Psychol. 2002;45(3):375-412.

36. Volterra V, Iverson J. When do modality factors affect the course of language acquisition? In: Reilly KEJ, editor. Language, gesture, and space. Hillsdale: Lawrence Erlbaum Associates, 1995. p. 371-90.

37. Iverson J, Tencer H, Lany J, Goldin-Meadow S. The relation between gesture and speech in congenitally blind and sighted language-learners. Journal of Nonverbal Behaviour. 2000;24(2):105-30.

38. Tomasello M, Carpenter M, Liszkowski U. A new look at infant pointing. Child Dev. 2007;78(3):705-22.

39. Obermeier C, Dolk T, Gunter TC. The benefits of gestures during communication, evidence from hearing and hearing-impaired individuals. Cortex. 2012;48(7):857-70.

40. Zaidman-Zait A, Dromi E. Analogous and distinctive patterns of prelinguistic communication in toddlers with and without hearing loss. J Speech Lang Hear Res. 2007;50(5):1166-80.

41. Goldin-Meadow S. Gesture, how our hands help us think. Cambridge: The Belknap Press of Harvard University Press; 2003.

42. Evans J, Alibali M, McNeil N. Divergence of embodied knowledge and verbal expression: Evidence from gesture and speech in children with specific language impairment. Language \& Cognitive Processes. 2001;16(2-3):309-31.

43. Martins I. Perturbações específicas do desenvolvimento da linguagem. Psicologia. 2002;16(1):27-50 